\title{
Learning to Avoid in Older Age
}

\author{
Michael J. Frank and Lauren Kong \\ University of Arizona
}

\begin{abstract}
The dopamine hypothesis of aging suggests that a monotonic dopaminergic decline accounts for many of the changes found in cognitive aging. The authors tested 44 older adults with a probabilistic selection task sensitive to dopaminergic function and designed to assess relative biases to learn more from positive or negative feedback. Previous studies demonstrated that low levels of dopamine lead to avoidance of those choices that lead to negative outcomes, whereas high levels of dopamine result in an increased sensitivity to positive outcomes. In the current study, age had a significant effect on the bias to avoid negative outcomes: Older seniors showed an enhanced tendency to learn from negative compared with positive consequences of their decisions. Younger seniors failed to show this negative learning bias. Moreover, the enhanced probabilistic integration of negative outcomes in older seniors was accompanied by a reduction in trial-to-trial learning from positive outcomes, thought to rely on working memory. These findings are consistent with models positing multiple neural mechanisms that support probabilistic integration and trial-to-trial behavior, which may be differentially impacted by older age.
\end{abstract}

Keywords: aging, reinforcement learning, dopamine, basal ganglia

As individuals age, they are faced with a variety of decisions, ranging from mundane (e.g., choosing an entrée on the dinner menu) to highly consequential (e.g., choosing a retirement plan). In making such choices, at least two different strategies can be employed to maximize benefit while minimizing risk. Individuals can opt for choices that have had positive outcomes in the past (e.g., "the roasted chicken was outstanding last time"), or they can avoid those decisions that have led to negative outcomes (e.g., "the shrimp made me sick last time"). The integration of these reinforcement outcomes over multiple experiences can lead to the development of a "gut feeling" for making choices without regard for any one individual memory. A possible mechanism for such reinforcement-based decision making is the neurotransmitter dopamine and its effects on synaptic plasticity. Dopamine plays a key role in the process of decision making and trial-and-error learning (Cools, Altamirano, \& D'Esposito, 2006; Frank, 2005; Frank, Moustafa, Haughey, Curran, \& Hutchison, 2007; Frank \& O'Reilly, 2006; Frank, Seeberger, \& O'Reilly, 2004; Gotham, Brown, \& Marsden, 1988; Knowlton, Mangels, \& Squire, 1996; Sevy et al., 2006; Shohamy, Myers, Geghman, Sage, \& Gluck, 2006).

A recent study further established the relationship between dopamine and reinforcement learning using a probabilistic selection task in which individuals were given feedback about their choices (Frank et al., 2004). We found that Parkinson's patients, who have depleted levels of dopamine, showed a greater tendency to avoid decisions leading to negative consequences than to seek

Michael J. Frank and Lauren Kong, Department of Psychology, University of Arizona.

We thank Karen Richardson and Tiffany Lupton-Stegall for help in administering cognitive tasks to participants and Greg Samanez Larkin for helpful comments and discussion.

Correspondence concerning this article should be addressed to Michael J. Frank, Department of Psychology, P.O. Box 210068, Tucson, AZ 857210068. E-mail: mfrank@u.arizona.edu positive outcomes. This pattern was reversed when patients took medications that elevated dopamine levels, causing patients to be more sensitive to positive than to negative outcomes. Thus, heightened dopamine levels are associated with seeking positive outcomes, whereas reduced dopamine levels are associated with avoiding negative outcomes. These findings support predictions from computational models of dopamine function (Brown, Bullock, \& Grossberg, 2004; Frank, 2005, 2006; O'Reilly \& Frank, 2006) and have been corroborated by dopamine manipulations in multiple other populations (Frank \& O'Reilly, 2006; Frank, Santamaria, O'Reilly, \& Willcutt, 2007; Waltz, Frank, Robinson, \& Gold, 2007). Moreover, these effects are consonant with previous studies that suggested that dopamine modulates probabilistic learning, conditional learning, and risky decision making in Parkinson's disease (Cools, 2006; Czernecki et al., 2002; Sevy et al., 2006; Shohamy, Myers, Grossman, Sage, \& Gluck, 2005; Shohamy et al., 2004; Vriezen \& Moscovitch, 1990).

Along with Parkinson's patients, older adults have also been reported to show reward-based learning deficits (Denburg, Recknor, Bechara, \& Tranel, 2006; Marschner et al., 2005; Mell et al., 2005). However, these findings are less consistent, with null effects reported as well (e.g., Kovalchik, Camerer, Grether, Plott, \& Allman, 2005; Samanez-Larkin et al., 2007; Wood, Busemeyer, Koling, Cox, \& Davis, 2005). Moreover, it is unclear whether deficits sometimes observed in reward-based learning tasks reflect a core learning deficit in the reinforcement learning system itself (i.e., subcortically) or whether they simply reflect working memory deficits known to accompany older age, associated with prefrontal deterioration, which could influence performance on such tasks.

It has been suggested that reinforcement learning impairments are the result of the simultaneous dopaminergic dysregulation that accompanies normal aging (Nieuwenhuis et al., 2002). Dopamine levels decline monotonically as individuals age (Martin, Palmer, Patlak, \& Calne, 1989; van Dyck et al., 2002; Volkow, Ding, et al., 1996; Volkow et al., 1998, 2000; Volkow, Wang, et al., 1996), and 
this decline in dopamine availability has been found to account for many other cognitive impairments that accompany normal aging (Bäckman et al., 2000; Bäckman, Nyberg, Lindenberger, Li, \& Farde, 2006; Kaasinen \& Rinne, 2002). A recent study demonstrated neuronal damage to dopaminergic cells themselves, similar to Parkinson's disease, in individuals older than 70 years of age (Kraytsberg et al., 2006).

If this dopamine hypothesis of aging is valid, we would expect older adults with more advanced dopamine depletion to show a pattern in the probabilistic selection task similar to that of nonmedicated Parkinson's patients (assuming that it is the low dopamine in Parkinson's, and not other disease-related factors, that drives the learning bias). In our Parkinson's studies, healthy senior controls were matched for age with Parkinson's patients $(M=65$ years) and, on average, learned equally well from positive and negative outcomes (Frank, Samanta, Moustafa, \& Sherman, 2007; Frank et al., 2004). We hypothesized that as healthy individuals get older (e.g., beyond 70; Kraytsberg et al., 2006), their decreasing dopamine levels would be associated with a greater propensity to avoid negative decision outcomes, together with reduced positive learning, compared with younger seniors. Thus, we predicted that younger old (Y-O) adults would show no learning bias one way or the other, whereas older old (O-O) adults would show a negative learning bias similar to that of Parkinson's patients.

These predictions are based on our models (e.g., Frank, 2005) and converging evidence from multiple populations and dopamine manipulations using the same task described below (Frank et al., 2004; Frank \& O'Reilly, 2006; Frank, Samanta, et al., 2007; Frank, Santamaria, et al., 2007; Frank, Woroch, \& Curran, 2005; Waltz et al., 2007). In the models, two basal ganglia pathways support go/no-go learning to choose actions that are probabilistically rewarded and to avoid those that are not rewarded. Dopamine bursts and dips that occur during positive and negative feedback (Schultz, 1998) drive go learning and no-go learning via effects on D1 and D2 dopamine receptors, which are relatively segregated in the two neural pathways. Thus, if dopamine levels are depleted, go learning should be impaired. However, according to this account, low levels of dopamine are actually beneficial for negative feedback learning. That is, for a person to learn from negative feedback, his or her dopamine levels must sufficiently decrease so that the no-go pathway can become more active (via D2 receptor disinhibition) and increase its synaptic weights. Notably, the no-go pathway is more excitable and plastic under conditions of dopamine depletion (Centonze et al., 2004; Day et al., 2006; Mallet, Ballion, Moine, \& Gonon, 2006). Supporting this account, researchers have shown that medications that elevate dopamine levels and/or tonically stimulate D2 receptors can prevent learning from negative outcomes (Frank, 2005; Frank, Samanta, et al., 2007; Frank et al., 2004; Cools et al., 2006). Thus, according to our models, relatively low baseline levels in older age, particularly in O-O adults, would make it more likely that all dopamine can be cleared from the synapse during pauses in dopamine cell firing, thereby enhancing no-go/avoidance learning (Frank, 2005; Frank et al., 2004).

Furthermore, because the D2 class of dopamine receptors are located in the no-go pathway and are particularly sensitive to small changes in dopamine levels (e.g., Frank \& O'Reilly, 2006), it is possible that the effects of older age might preferentially affect no-go learning. Thus, in healthy older adults, there might be sufficient dopamine available to support D1-dependent probabalistic go learning, which would be impacted only by more extreme dopamine depletion. Although some (but not all) studies discussed above have shown reward learning deficits in older age, these typically pertain to trial-to-trial learning of reward contingencies, as in the Iowa Gambling Task (Denburg et al., 2006; Wood et al., 2005), which may tax working memory to a greater extent in older adults. Thus, O-O adults might show impairments in the rapid trial-to-trial learning from positive outcomes because of working memory deficits but show relatively enhanced probabilistic integration of negative outcomes in the long run because of dopamine reductions in the basal ganglia. Such a prediction does not follow from more general theories of cognitive deficits in older age but, rather, relies on the notion that probabalistic go/no-go learning and rapid trial-to-trial learning are distinct cognitive and neural processes (Frank \& Claus, 2006; Frank, Moustafa, et al., 2007), which may be differentially sensitive to older age.

\section{Method}

\section{Sample}

We tested 44 older adults ( 24 women, 20 men) between the ages of 60 and 83 years $(M=72, S D=6.0)$. Mean years of education were 16.3 years $(S D=2.6)$, and mean number of errors in the North American Adult Reading Test (a measure of verbal IQ; Blair \& Spreen, 1989) was $13.7(S D=5.9)$. Participants were selected from a pool of healthy, community-dwelling older adults and received monetary compensation for their participation. All participants were currently in good health and reported no depression, dementia, or previous neurological problems that might impair cognitive function. One participant's data were discarded because of a computer crash.

\section{Procedure}

Participants were seated in front of a computer screen in a lighted room and viewed pairs of visual stimuli that were not easily verbalized (Japanese Hiragana characters). These stimuli were presented in black on a white background, in 72-point font. The participants pressed keys on the left or right side of the keyboard, depending on which stimulus they chose to be "correct." Visual feedback was provided following each choice ("Correct!" printed in blue or "Incorrect" printed in red). If no response was made within $4 \mathrm{~s}$, the words "no response detected" were displayed in red.

Participants were tested with the Probabilistic Selection Task (Frank et al., 2004). Three different stimulus pairs (AB, CD, EF) were presented in random order (see Figure 1A), with the assignment of Hiragana character to Stimulus Elements A-F counterbalanced across subjects. Feedback followed the choice to indicate whether it was correct or incorrect, but this feedback was probabilistic. Choosing Stimulus A led to correct (positive) feedback in $80 \%$ of $\mathrm{AB}$ trials, whereas choosing Stimulus B led to incorrect (negative) feedback in these trials. $\mathrm{CD}$ and $\mathrm{EF}$ pairs were less reliable: Stimulus $\mathrm{C}$ was correct in $70 \%$ of $\mathrm{CD}$ trials, whereas $\mathrm{E}$ was correct in $60 \%$ of $\mathrm{EF}$ trials. Over the course of training, participants learned to choose Stimuli A, C, and E more often than B, D, or F.

We enforced a performance criterion (evaluated after each training block of 60 trials) to ensure that all participants were at the same performance level before advancing to test. Because of the different probabilistic structure of each stimulus pair, we used a 

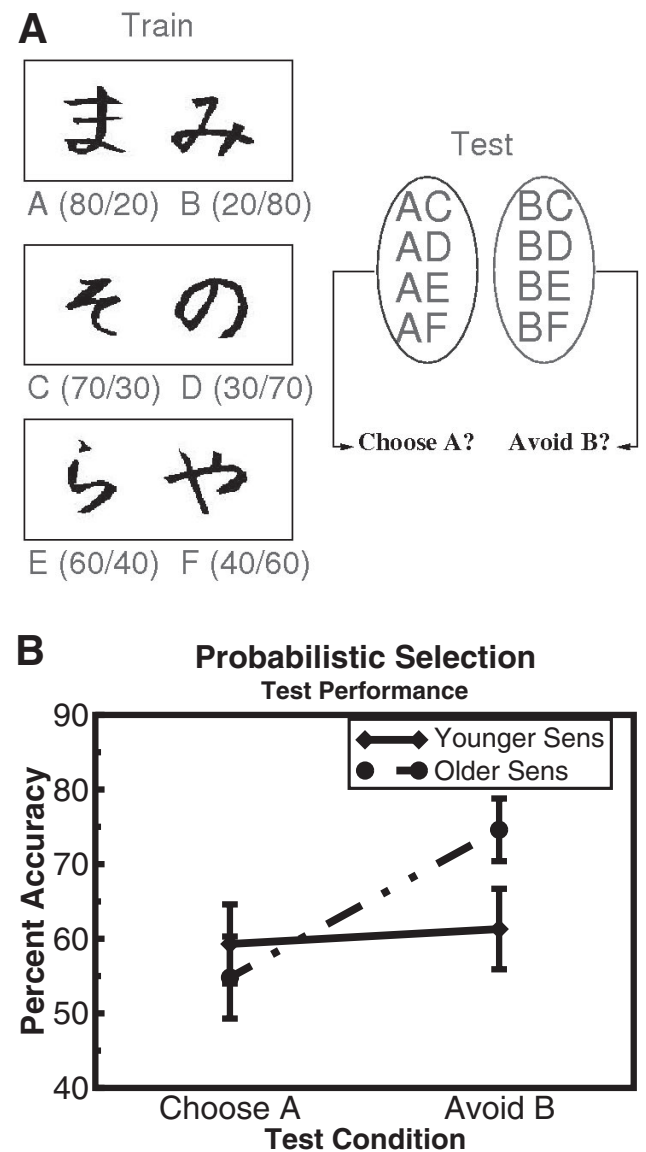

Figure 1. A: Example stimulus pairs (Hiragana characters), designed to minimize verbal encoding. The frequency of positive feedback for each choice is shown. B: Novel test-pair performance in younger and older sens (seniors). Error bars reflect standard error of the mean.

different criterion for each $(65 \% \mathrm{~A}$ in $\mathrm{AB}, 60 \% \mathrm{C}$ in $\mathrm{CD}, 50 \% \mathrm{E}$ in $\mathrm{EF}$ ). (In the EF pair, stimulus $\mathrm{E}$ is correct $60 \%$ of the time, but this is particularly difficult to learn. We therefore used a $50 \%$ criterion for this pair simply to ensure that if participants happened to like Stimulus F at the outset, they nevertheless had to learn that this bias was not going to consistently work.) The participant advanced to the test session if all these criteria were met or after six blocks (360 trials) of training.

Participants were subsequently tested with the same training pairs, in addition to all novel combinations of stimuli, in random sequence. Prior to the test phase, they were given the following instructions:

It's time to test what you've learned! During this set of trials you will NOT receive feedback ("Correct" or "Incorrect") to your responses. If you see new combinations of symbols in the test, please choose the symbol that "feels" more correct based on what you learnt during the training sessions. If you're not sure which one to pick, just go with your gut instinct!

Each test pair was presented four times for a maximum of $4 \mathrm{~s}$ duration, and no feedback was provided.
Note that learning to choose A over B could be accomplished either by learning that A leads to positive feedback or that B leads to negative feedback (or both). To evaluate whether participants learned more about positive or negative outcomes of their decisions, we subsequently tested them with novel combinations of stimulus pairs involving either an $\mathrm{A}(\mathrm{AC}, \mathrm{AD}, \mathrm{AE}, \mathrm{AF})$ or a $\mathrm{B}$ (BC, BD, BE, BF); no feedback was provided. If participants had learned more from positive feedback, they should have reliably chosen Stimulus A in all novel test pairs in which it was present. On the other hand, if they learned more from negative feedback, they should have more reliably avoided Stimulus B. Consistent with this depiction, error-related brain activity was enhanced in young participants who learned to avoid B, compared with those who more successfully chose A (Frank et al., 2005).

\section{Results}

General linear model regressions revealed that avoid-B performance significantly improved with age, $F(1,41)=5.2, p=.027$, $R^{2}=0.11$, but there was no effect of age on choose-A performance, $F(1,41)=0.37$. The relative within-subjects tendency to learn more from negative than from positive outcomes of decisions also increased with age, but this effect did not quite reach significance, $F(1,41)=$ $3.4, p=.07, R^{2}=0.08$. These learning biases were revealed despite no effect of age on the number of training trials needed to reach criteria, accuracy during the training phase, or test-phase performance on the studied $\mathrm{AB}$ training pair (all $p \mathrm{~s}>.3$ ).

To further investigate these learning biases, we analyzed trial-totrial behavior as a function of error feedback during the first block of training. Win-stay behavior was defined as the percentage of trials following positive feedback in which participants chose the same stimulus (e.g., choosing A after it had been rewarded the last time it appeared). Lose-shift behavior was defined as the percentage of trials following negative feedback in which participants avoided selecting the same stimulus (e.g., avoiding B after it led to negative feedback the last time it appeared). These analyses are thought to reflect sensitivity to the recency of positive and negative outcomes, which may depend more on working memory than on the probabilistic test-phase results described above (Frank, Moustafa, et al., 2007). Corroborating the test-phase results, we found that the difference between win-stay and lose-shift performance increased with age, $F(1$, $41)=4.24, p=.045, R^{2}=0.1$, such that older participants were less likely to win-stay and more likely to lose-shift. However, this was due to a significant negative relationship between age and win-stay performance, $F(1,41)=4.9, p=.03, R^{2}=0.11$, and a nonsignificant tendency for lose-shift to increase with age $(p>.18)$. Thus, older adults were impaired in trial-to-trial learning from rewarding outcomes but showed enhanced probabilistic learning from negative outcomes.

In our previous studies with Parkinson's patients, healthy senior controls learned equally well from the positive and negative consequences of their decisions (Frank, Samanta, et al., 2007; Frank et al., 2004). It is critical to note that these participants were younger than those in the current study who showed a negative bias. To determine the age constraints where the negative biases become apparent, we used a median split to divide seniors in the current study into two groups. These results complement the correlational (continuous) measure described above and show that negative biases are consistently observed only in the older half of partici- 
pants. O-O adults were, on average, 10 years older $(M=77, S D=$ $3.0)$ than the Y-O adults $(M=67, S D=3.5)$. There were no differences between these age groups in demographic variables, including years of education ( $M=16.0 \mathrm{vs.} 16.5$ years for younger and older seniors, respectively), $F(1,41)=0.3$, and an estimate of verbal IQ using the North American Adult Reading Test (Blair \& Spreen, 1989; $M=13.8$ vs. 13.6 errors for Y-O adults and O-O adults, respectively), $F(1,41)=0.01$. There were also no group differences in overall test-phase performance in the probabilistic selection task, $F(1,41)=1.9$, ns. Nevertheless, the interaction between age group and test-pair condition (choose-A and avoid-B) was significant (see Figure $1 \mathrm{~B}$ ), $F(1,41)=4.7, p=.036$. O-O seniors were better at negative than positive learning, $F(1,21)=$ $16.1, p=.0006$, whereas $\mathrm{Y}-\mathrm{O}$ seniors did not differ in these measures (see Figure 2), $F(1,20)=0.06$. There was also a marginal interaction between age group and win-stay versus loseshift performance during training (see Figure 3 ), $F(1,41)=3.3$, $p=.078$.

A potential alternative account of the data is that, rather than showing differences in avoidance learning, perhaps O-O participants are more likely than their Y-O counterparts to optimize, rather than match, responding (e.g., Estes, 1961; Shanks, Tunney, \& McCarthy, 2002). Optimizing is characterized by always choosing the stimulus with greatest reward probability, whereas matching reflects a tendency to allocate behavioral choices in proportion to the probabilities. It is in principle possible that, because of loss aversion, participants are more likely to optimize for negative test pairs (e.g., always avoid B) than for positive pairs, in which they might be more likely to match. If this were the case, the enhanced avoid-B performance in older participants could reflect a greater propensity to optimize with age, rather than better negative feedback learning per se.

To address this possibility, we divided test pairs into those that would be more or less likely to produce matching. Specifically, in high-conflict choices, reinforcement values are similar between the two stimuli in novel test pairs (i.e., win/win or lose/lose decisions, e.g., 80 vs. 70 or 20 vs. 30; Frank, Samanta, et al., 2007; Frank, Woroch, \& Curran, 2005). Thus, if one were to probability match, it would be more apparent in these high-conflict choices, compared with low-conflict choices (e.g., 80 vs. 30). In turn, this would imply that $\mathrm{O}-\mathrm{O}$ adults' better avoid-B performance would be

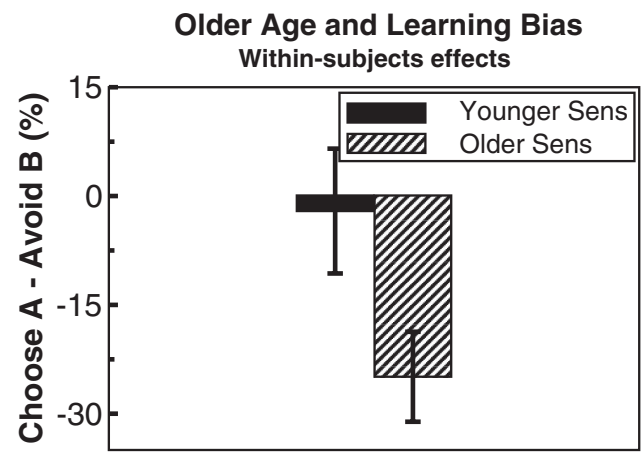

Figure 2. Relative within-subjects biases to learn more from positive relative to negative reinforcement across age groups. Sens $=$ seniors. Error bars reflect standard error of the mean.

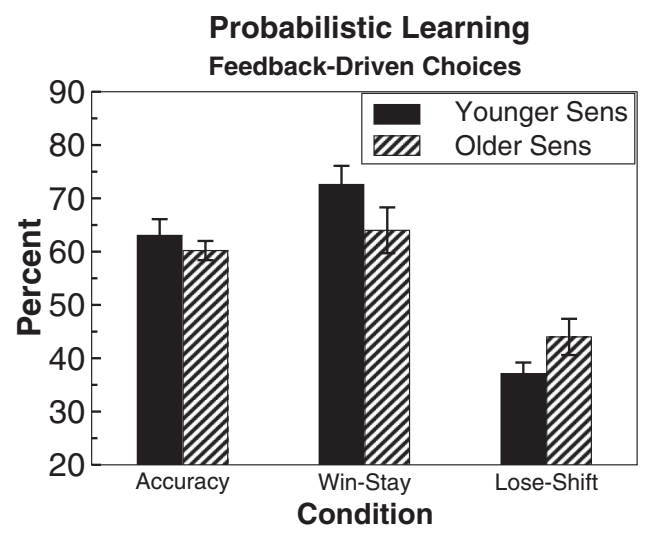

Figure 3. Training performance and trial-to-trial learning from positive and negative feedback (win-stay and lose-shift, respectively). Older sens (seniors) were more likely to display lose-shift than win-stay behavior relative to younger seniors. Error bars reflect standard error of the mean.

particularly evident in high-conflict choices that would produce matching in $\mathrm{Y}-\mathrm{O}$ adults.

Contrary to this account, avoid-B performance was reliably better in O-O than in Y-O seniors, regardless of conflict, and was thus not sensitive to likelihood of matching. To address this statistically, we performed a 2 (conflict) $\times 2$ (choose-A vs. avoid-B) $\times 2$ (age group) repeated measures analysis of variance. There was a main effect of age group on avoid-B versus choose-A, $F(1,41)=4.8, p=.03$. There was also a main effect of conflict, $F(1,41)=5.2, p=.027$, such that performance was better overall in low-conflict conditions (or, alternatively, participants were more likely to probability match in high-conflict choices). But critically, this conflict effect did not interact with either age group, $F(1,41)=0.2$, or positive versus negative condition, $F(1,41)=0.1$. O-O seniors avoided Stimulus B in $84 \%$ of low-conflict negative conditions, whereas Y-O seniors did so only $67 \%$ of the time; this difference was significant, $F(1,41)=4.6, p=$ .037. Similarly, O-O seniors avoided B in $76 \%$ of high-conflict choices, whereas Y-O seniors did so in just $55 \%$ of choices, $F(1$, $41)=6.6, p=.01$. In contrast, $\mathrm{Y}-\mathrm{O}$ seniors were numerically (but insignificantly) better than $\mathrm{O}-\mathrm{O}$ seniors at positive (choose-A) conditions for both low-conflict (64\% vs. 58\%) and high-conflict choices ( $54 \%$ vs. $52 \%$ ). Finally, the three-way interaction between age, conflict, and positive/negative condition was not significant, $F(1,41)=$ 0.01 . These results confirm an overall avoidance bias with older age, which cannot be easily explained by differences in optimizing versus matching.

\section{Discussion}

Individuals tend to become more conservative with age (e.g., Botwinick, 1969). Indeed, older adults become more cautious with their decisions and take fewer risks (Deakin, Aitken, Robbins, \& Sahakian, 2004; Okun \& Vesta, 1976; Wallach \& Kogan, 1961). It is possible that this risk-avoidant behavior arises from reduced levels of the neurotransmitter dopamine, leading to a no-go bias to avoid potential negative outcomes in the basal ganglia decisionmaking system. In the present study, it was found that older seniors learn better from the negative than the positive outcomes of their decisions in a probabilistic task. This avoidance bias is likely 
dopamine related, as this same avoidance bias was found in Parkinson's patients with low dopamine levels and in healthy younger adults taking drugs that reduce dopamine levels (Frank \& O'Reilly, 2006; Frank et al., 2004). Further, drugs that elevate dopamine levels disrupt avoidance learning in this task and in others (Cools et al., 2006; Frank \& O'Reilly, 2006; Frank, Santamaria, et al., 2007; Frank et al., 2004). Moreover, the increase in avoidance bias in adults older than roughly 70 years is in accordance with a recent study showing that this age demarcation is associated with dramatic changes in dopamine neuronal damage (Kraytsberg et al., 2006). The younger seniors showed a numerical trend to be more sensitive to probabilistic positive reinforcement, but this pattern failed to reach significance.

These findings and interpretation are in accordance with the dopamine hypothesis of cognitive aging, which suggests that many of the cognitive impairments that accompany normal aging are due, at least in part, to a simultaneous decline in dopamine availability (Bäckman et al., 2000; Braver \& Barch, 2002; Braver et al., 2001; Kaasinen \& Rinne, 2002; Volkow et al., 1998). Note that alternative accounts of reinforcement learning posit that whereas increases in dopamine support positive reinforcement learning, increases in serotonin support aversive learning (e.g., Daw, Kakade, \& Dayan, 2002). Supporting this notion, researchers have found that drugs that increase serotonin concentration have been shown to enhance negative feedback sensitivity in a probabilistic learning task (Chamberlain et al., 2006). However, the reported effects influenced trial-to-trial sensitivity to negative outcomes, and it is possible that serotonin can enhance working memory for recent negative outcomes (see Frank \& Claus, 2006, for discussion). Moreover, it is unclear how a serotonin-based account could explain our observed data. In addition to dopamine, serotonin levels also decrease both in healthy aging (van Dyck et al., 2000; Versijpt et al., 2003; Yamamoto et al., 2002) and in Parkinson's disease (e.g., Kish, 2003). Given that the above-mentioned hypothesis suggests that increases in serotonin support aversive learning, these age-related serotonin depletions would predict, if anything, worse avoidance learning - the opposite of what we have found in both older seniors and Parkinson's patients. Nevertheless, future combined imaging and probabilistic learning studies are required to more conclusively establish a dopaminergic basis for the observed avoidance bias in older age.

On the surface, our study contrasts with a recent behavioral and neuroimaging study in which older adults showed reduced, rather than enhanced, neural sensitivity to losses (Samanez-Larkin et al., 2007). However, in that study, the observed differences between younger and older adults pertained to the anticipation of impending losses that would occur seconds later; no differences were seen in response to negative outcomes themselves. Moreover, the reduced loss anticipation reflected a lack of discrimination between different magnitudes of negative outcomes, which correlated with participants' self-reported affective reaction to the stimuli. In contrast, our study focused on the ability to integrate the probability of negative outcomes to support avoidance of the most negative stimulus in the long run. We have argued that this learning is relatively implicit and depends on the basal ganglia, whereas prefrontal areas are necessary for representing reinforcement magnitudes over delays (Frank \& Claus, 2006; Frank, Moustafa, et al., 2007) in a more conscious format that would be accessible for self-report. Although various studies report ventral striatal activity encoding magnitudes of gains or losses, this may reflect top-down input from frontal regions; our claim is simply that the basal ganglia are biased to learn about reinforcement frequency over multiple trials but that they can also receive information about reinforcement magnitudes in any one trial from areas including frontal cortex and amygdala (Frank \& Claus, 2006). It is noteworthy in this regard that although older adults showed enhanced probabilistic negative learning in this study, they did not show significantly greater lose-shift performance on a trial-to-trial basis, a function that we attribute to the prefrontal system. Supporting this notion, researchers have found that a gene that codes for prefrontal dopamine predicts lose-shift behavior but not probabilistic avoidance learning in our task, whereas a gene coding for striatal D2 receptor density is associated with probabilistic avoidance learning but not lose-shift (Frank, Moustafa, et al., 2007). Future research should assess more directly whether there is dissociation between prefrontal-dependent and basal gangliadependent loss of sensitivity in older age, within the same group of participants.

How do our findings relate to other cognitive deficits generally observed in older age? Tasks that require individuals to modify their behavior in response to both positive and negative feedback, such as a set-shifting, probabilistic classification, or gambling tasks, are typically impaired in older adults (Chasseigne et al., 2004; Denburg et al., 2006; Rhodes, 2004). As with the probabilistic selection task used in this study, performance on these tasks depends on how well an individual can learn to make choices that lead to positive outcomes and to avoid those that lead to negative outcomes. In set-shifting tasks, individuals are required to shift their attention among stimulus dimensions, relying on reinforcement to guide their decisions. A potential explanation is that shifting deficits in older age may reflect exaggerated learning to ignore task-irrelevant (negative) information over multiple trials during the initial phase of the task, making it more difficult to then pay attention to this information when it subsequently becomes relevant (for more explicit discussion of this account, see Frank and O'Reilly, 2006). This pattern of learned irrelevance can also explain set-shifting deficits in Parkinson's disease patients (Gauntlett-Gilbert, Roberts, \& Brown, 1999; Owen et al., 1993; Slabosz et al., 2006), who seem to have more difficulty attending to previously irrelevant information than ignoring previously relevant information (e.g., Slabosz et al., 2006).

Similarly, probabilistic classification tasks use trial-and-error feedback to guide individuals into making the correct predictions on the basis of probabilistic patterns. Older adults sometimes show impairments on these tasks, which may stem from declining dopamine levels and/or impaired working memory. The current probabilistic selection task did not produce overall learning deficits in the oldest age group, but this might reflect the fact that this task can be successfully solved via negative feedback learning, whereas in more complex tasks, an integration of both positive and negative feedback is critical. Further, older adults in the current study did show impairment in trial-to-trial learning from rewarding outcomes (win-stay), which could reflect a working memory deficit and may relate to the previously documented impairments in reward learning. Alternatively, older adults might also show a deficit in the slow integration of reward signals across trials, even though this was not observed in the present study. Reduced dynamic range of the dopamine system, as in Parkinson's disease, 
has been shown to account for impaired probabilistic classification learning in the same computational model and, with the same parameters, used to simulate enhanced avoidance learning (Frank, 2005; Frank et al., 2004). This interpretation is consistent with observed impairments in probabilistic learning tasks in patients with dopaminergic dysfunction, such as Parkinson's and Huntington's disease patients (Cools, 2006; Czernecki et al., 2002; Frank, 2005; Lawrence et al., 1996; Lawrence, Sahakian, Rogers, Hodges, \& Robbins, 1999; Perretta, Pari, \& Beninger, 2005; Shohamy et al., 2004).

\section{Conclusion}

Dopamine plays an important role in learning from the outcomes of one's previous experiences. As individuals age, they tend to be more cautious in their decision making. This risk-avoidant behavior may be due to an enhanced ability to avoid negative stimuli. Although our probability learning paradigm is clearly not equivalent to real-world conservative behavior, it does allow assessment of an implicit bias to learn to avoid negative events, compared with learning to approach positive stimuli. We found that increasing age in older adults is associated with a greater bias to learn to avoid negative events. Our findings suggest that this risk-avoidant bias increases with age and may be a sequela of the dopaminergic changes that accompany normal aging.

\section{References}

Bäckman, L., Ginovart, N., Dixon, R. A., Wahlin, T. R., Wahlin, A., Halldin, C., \& Farde, L. (2000). Age-related cognitive deficits mediated by changes in the striatal dopamine system. American Journal of Psychiatry, 157(4), 635-637.

Bäckman, L., Nyberg, L., Lindenberger, U., Li, S.-C., \& Farde, L. (2006). The correlative triad among aging, dopamine, and cognition: Current status and future prospects. Neuroscience and Biobehavioral Reviews, 30(6), 791-807.

Blair, J. R., \& Spreen, O. (1989). Predicting premorbid IQ: A revision of the National Adult Reading Test. Clinical Neuropsychologist, 3, 129-136.

Botwinick, J. (1969). Disinclination to venture versus cautiousness in responding: Age differences. Journal of Genetic Psychology, 115, 55-62.

Braver, T. S., \& Barch, D. M. (2002). A theory of cognitive control, aging cognition, and neuromodulation. Neuroscience and Biobehavioral Reviews, 26(7), 809-817.

Braver, T. S., Barch, D. M., Keys, B. A., Carter, C. S., Cohen, J. D., Kaye, J. A., et al. (2001). Context processing in older adults: Evidence for a theory relating cognitive control to neurobiology in healthy aging. Journal of Experimental Psychology: General, 130, 746-763.

Brown, J. W., Bullock, D., \& Grossberg, S. (2004). How laminar frontal cortex and basal ganglia circuits interact to control planned and reactive saccades. Neural Networks, 17, 471-510.

Centonze, D., Usiello, A., Costa, C., Picconi, B., Erbs, E., Bernardi, G., et al. (2004). Chronic haloperidol promotes corticostriatal long-term potentiation by targeting dopamine D2L receptors. Journal of Neuroscience, 24, 8214-8222.

Chamberlain, S. R., Muller, U., Blackwell, A. D., Clark, L., Robbins, T. W., \& Sahakian, B. J. (2006, February 10). Neurochemical modulation of response inhibition and probabilistic learning in humans. Science, 311(5762), 861-863.

Chasseigne, G., Ligneau, C., Grau, S., Gall, A. L., Roque, M., \& Mullet, E. (2004). Aging and probabilistic learning in single- and multiple-cue tasks. Experimental Aging Research, 30(1), 23-45.

Cools, R. (2006). Dopaminergic modulation of cognitive function- implications for L-DOPA treatment in Parkinson's disease. Neuroscience and Biobehavioral Reviews, 30(1), 1-23.

Cools, R., Altamirano, L., \& D’Esposito, M. (2006). Reversal learning in Parkinson's disease depends on medication status and outcome valence. Neuropsychologia, 44, 1663-1673.

Czernecki, V., Pillon, B., Houeto, J. L., Pochon, J. B., Levy, R., \& Dubois, B. (2002). Motivation, reward, and Parkinson's disease: Influence of dopatherapy. Neuropsychologia, 40, 2257-2267.

Daw, N. D., Kakade, S., \& Dayan, P. (2002). Opponent interactions between serotonin and dopamine. Neural Networks, 15, 603-616.

Day, M., Wang, Z., Ding, J., An, X., Ingham, C. A., Shering, A. F., et al. (2006). Selective elimination of glutamatergic synapses on striatopallidal neurons in Parkinson disease models. Nature Neuroscience, 9(2), 251-259.

Deakin, J., Aitken, M., Robbins, T., \& Sahakian, B. J. (2004). Risk taking during decision-making in normal volunteers changes with age. Journal of the International Neuropsychological Society, 10(4), 590-598.

Denburg, N. L., Recknor, E. C., Bechara, A., \& Tranel, D. (2006). Psychophysiological anticipation of positive outcomes promotes advantageous decision-making in normal older persons. International Journal of Psychophysiology, 61(1), 19-25.

Estes, W. K. (1961). A descriptive approach to the dynamics of choice behavior. Behavioral Science, 6, 177-184.

Frank, M. J. (2005). Dynamic dopamine modulation in the basal ganglia: A neurocomputational account of cognitive deficits in medicated and non-medicated parkinsonism. Journal of Cognitive Neuroscience, 17, 51-72.

Frank, M. J. (2006). Hold your horses: A dynamic computational role for the subthalamic nucleus in decision making. Neural Networks, 19, $1120-1136$

Frank, M. J., \& Claus, E. D. (2006). Anatomy of a decision: Striatoorbitofrontal interactions in reinforcement learning, decision making, and reversal. Psychological Review, 113(2), 300-326.

Frank, M. J., Moustafa, A. A., Haughey, H., Curran, T., \& Hutchison, K. (2007). Genetic triple dissociation reveals multiple roles for dopamine in reinforcement learning. Proceedings of the National Academy of Sciences, USA, 104, 16311-16316.

Frank, M. J., \& O'Reilly, R. C. (2006). A mechanistic account of striatal dopamine function in human cognition: Psychopharmacological studies with cabergoline and haloperidol. Behavioral Neuroscience, 120, $497-$ 517.

Frank, M. J., Samanta, J., Moustafa, A. A., \& Sherman, S. J. (2007, October 25). Hold your horses: Impulsivity, deep brain stimulation and medication in parkinsonism. Science, 318, 1309-1312.

Frank, M. J., Santamaria, A., O'Reilly, R. C., \& Willcutt, E. (2007). Testing computational models of dopamine and noradrenaline dysfunction in attention deficit/hyperactivity disorder. Neuropsychopharmacology, 32, 1583-1599.

Frank, M. J., Seeberger, L. C., \& O'Reilly, R. C. (2004, December 10). By carrot or by stick: Cognitive reinforcement learning in parkinsonism. Science, 306, 1940-1943.

Frank, M. J., Woroch, B. S., \& Curran, T. (2005). Error-related negativity predicts reinforcement learning and conflict biases. Neuron, 47, 495501.

Gauntlett-Gilbert, J., Roberts, R. C., \& Brown, V. J. (1999). Mechanisms underlying attentional set-shifting in Parkinson's disease. Neuropsychologia, 37, 605-616.

Gotham, A. M., Brown, R. G., \& Marsden, C. D. (1988). "Frontal" cognitive function in patients with Parkinson's disease "on" and "off" levodopa. Brain, 111, 299-321.

Kaasinen, V., \& Rinne, J. O. (2002). Functional imaging studies of dopamine system and cognition in normal aging and Parkinson's disease. Neuroscience and Biobehavioral Reviews, 26(7), 785-793.

Kish, S. J. (2003). Biochemistry of Parkinson's disease: Is a brain seroto- 
nergic deficiency a characteristic of idiopathic Parkinson's disease? Advances in Neurology, 91, 39-49.

Knowlton, B. J., Mangels, J. A., \& Squire, L. R. (1996, September 6). A neostriatal habit learning system in humans. Science, 273, 1399.

Kovalchik, S., Camerer, C. F., Grether, D. M., Plott, C. R., \& Allman, J. M. (2005). Aging and decision making: A comparison between neurologically healthy elderly and young individuals. Journal of Economic Behavior \& Organization, 58, 79-94.

Kraytsberg, Y., Kudryavtseva, E., McKee, A. C., Geula, C., Kowall, N. W., \& Khrapko, K. (2006). Mitochondrial DNA deletions are abundant and cause functional impairment in aged human substantia nigra neurons. Nature Genetics, 38(5), 518-520.

Lawrence, A. D., Sahakian, B. J., Hodges, J. R., Rosser, A. E., Lange, K. W., \& Robbins, T. W. (1996). Executive and mnemonic functions in early Huntington's disease. Brain, 119(5), 1633-1645.

Lawrence, A. D., Sahakian, B. J., Rogers, R. D., Hodges, J. R., \& Robbins, T. W. (1999). Discrimination, reversal, and shift learning in Huntington's disease: Mechanisms of impaired response selection. Neuropsychologia, 37, 1359-1374.

Mallet, N., Ballion, B., Moine, C. L., \& Gonon, F. (2006). Cortical inputs and GABA interneurons imbalance projection neurons in the striatum of parkinsonian rats. Journal of Neuroscience, 26(14), 3875-3884.

Marschner, A., Mell, T., Wartenburger, I., Villringer, A., Reischies, F. M., \& Heekeren, H. R. (2005). Reward-based decision-making and aging. Brain Research Bulletin, 67(5), 382-390.

Martin, W. R., Palmer, M. R., Patlak, C. S., \& Calne, D. B. (1989). Nigrostriatal function in humans studied with positron emission tomography. Annals of Neurology, 26(4), 535-542.

Mell, T., Heekeren, H. R., Marschner, A., Wartenburger, I., Villringer, A., \& Reischies, F. M. (2005). Effect of aging on stimulus-reward association learning. Neuropsychologia, 43(4), 554-563.

Nieuwenhuis, S., Ridderinkhof, K. R., Talsma, D., Coles, M. G., Holroyd, C. B., Kok, A., \& Van Der Molen, M. W. (2002). A computational account of altered error processing in older age: Dopamine and the error-related negativity. Cognitive, Affective, \& Behavioral Neuroscience, 2, 19-36.

O’Reilly, R. C., \& Frank, M. J. (2006). Making working memory work: A computational model of learning in the prefrontal cortex and basal ganglia. Neural Computation, 18, 283-328.

Okun, M. A., \& Vesta, F. J. D. (1976). Cautiousness in adulthood as a function of age and instructions. Journal of Gerontology, 31(5), 571-576.

Owen, A. M., Roberts, A. C., Hodges, J. R., Summers, B. A., Polkey, C. E., \& Robbins, T. W. (1993). Contrasting mechanisms of impaired attentional set-shifting in patients with frontal lobe damage or Parkinson's disease. Brain, 116, 1159-1175.

Perretta, J. G., Pari, G., \& Beninger, R. J. (2005). Effects of Parkinson disease on two putative nondeclarative learning tasks: Probabilistic classification and gambling. Cognitive and Behavioral Neurology, 18(4), 185-192.

Rhodes, M. G. (2004). Age-related differences in performance on the Wisconsin Card Sorting Test: A meta-analytic review. Psychology and Aging, 19(3), 482-494.

Samanez-Larkin, G. R., Gibbs, S. E. B., Khanna, K., Nielsen, L., Carstensen, L. L., \& Knutson, B. (2007). Anticipation of monetary gain but not loss in healthy older adults. Nature Neuroscience, 10(6), 787-791.

Schultz, W. (1998). Predictive reward signal of dopamine neurons. Journal of Neurophysiology, 80, 1-27.

Sevy, S., Hassoun, Y., Bechara, A., Yechiam, E., Napolitano, B., Burdick, K., et al. (2006). Emotion-based decision-making in healthy subjects: Short-term effects of reducing dopamine levels. Psychopharmacology (Berlin), 188(2), 228-235.

Shanks, D. R., Tunney, R. J., \& McCarthy, J. D. (2002). A re-examination of probability matching and rational choice. Journal of Behavioral Decision Making, 15, 233-250.
Shohamy, D., Myers, C. E., Geghman, K. D., Sage, J., \& Gluck, M. A. (2006). L-dopa impairs learning, but spares generalization, in Parkinson's disease. Neuropsychologia, 44(5), 774-784.

Shohamy, D., Myers, C. E., Grossman, S., Sage, J., \& Gluck, M. A. (2005). The role of dopamine in cognitive sequence learning: Evidence from Parkinson's disease. Behavioural Brain Research, 156(2), 191-199.

Shohamy, D., Myers, C. E., Grossman, S., Sage, J., Gluck, M. A., \& Poldrack, R. A. (2004). Cortico-striatal contributions to feedback-based learning: Converging data from neuroimaging and neuropsychology. Brain, 127, 851-859.

Slabosz, A., Lewis, S. J. G., Smigasiewicz, K., Szymura, B., Barker, R. A., \& Owen, A. M. (2006). The role of learned irrelevance in attentional set-shifting impairments in Parkinson's disease. Neuropsychology, 20(5), 578-588.

van Dyck, C. H., Malison, R. T., Seibyl, J. P., Laruelle, M., Klumpp, H., Zoghbi, S. S., et al. (2000). Age-related decline in central serotonin transporter availability with [(123)I]beta-CIT SPECT. Neurobiology of Aging, 21(4), 497-501.

van Dyck, C. H., Seibyl, J. P., Malison, R. T., Laruelle, M., Zoghbi, S. S., Baldwin, R. M., \& Innis, R. B. (2002). Age-related decline in dopamine transporters: Analysis of striatal subregions, nonlinear effects, and hemispheric asymmetries. American Journal of Geriatric Psychiatry, 10(1), $36-43$.

Versijpt, J., Laere, K. J. V., Dumont, F., Decoo, D., Vandecapelle, M., Santens, P., et al. (2003). Imaging of the 5-HT2A system: Age-, gender-, and Alzheimer's disease-related findings. Neurobiology of Aging, 24(4), $553-561$.

Volkow, N. D., Ding, Y. S., Fowler, J. S., Wang, G. J., Logan, J., Gatley, S. J., et al. (1996). Dopamine transporters decrease with age. Journal of Nuclear Medicine, 37, 554-559.

Volkow, N. D., Gur, R. C., Wang, G.-J., Fowler, J. S., Moberg, P. J., Ding, Y.-S., et al. (1998). Association between decline in brain dopamine activity with age and cognitive and motor impairment in healthy individuals. American Journal of Psychiatry, 155, 344-349.

Volkow, N. D., Logan, J., Fowler, J. S., Wang, G., Gur, R. C., Wong, C., et al. (2000). Association between age-related decline in brain dopamine activity and impairment in frontal and cingulate metabolism. American Journal of Psychiatry, 157(1), 75-80.

Volkow, N. D., Wang, G. J., Fowler, J. S., Logan, J., Gatley, S. J., MacGregor, R. R., et al. (1996). Measuring age-related changes in DA D2 receptors with $[\mathrm{C}]$ raclopride and with $[\mathrm{F}] \mathrm{N}$-methylspiroperidol. Psychiatry Research, 67, 11-16.

Vriezen, E. R., \& Moscovitch, M. (1990). Memory for temporal order and conditional associative-learning in patients with Parkinson's disease. Neuropsychologia, 28, 1283-1293.

Wallach, M. A., \& Kogan, N. (1961). Aspects of judgment and decision making: Interrelationships and changes with age. Behavioral Science, 6 , 23-36.

Waltz, J. A., Frank, M. J., Robinson, B. M., \& Gold, J. M. (2007). Selective reinforcement learning deficits in schizophrenia support predictions from computational models of striatal-cortical dysfunction. Biological Psychiatry, 62, 756-764.

Wood, S., Busemeyer, J., Koling, A., Cox, C. R., \& Davis, H. (2005). Older adults as adaptive decision makers: Evidence from the Iowa Gambling Task. Psychology and Aging, 20(2), 220-225.

Yamamoto, M., Suhara, T., Okubo, Y., Ichimiya, T., Sudo, Y., Inoue, M., et al. (2002). Age-related decline of serotonin transporters in living human brain of healthy males. Life Sciences, 71(7), 751-757.

Received August 7, 2007

Revision received November 29, 2007 Accepted December 3, 2007 